\title{
The Prefrontal Cortex Achieves Inhibitory Control by Facilitating Subcortical Motor Pathway Connectivity
}

\author{
(1)Charlotte L. Rae, ${ }^{1,2}$ Laura E. Hughes, ${ }^{1,2}$ Michael C. Anderson, ${ }^{1,3}$ and $\oplus$ James B. Rowe ${ }^{1,2,3}$ \\ ${ }^{1} \mathrm{MRC}$ Cognition and Brain Sciences Unit, Cambridge, CB2 7EF, United Kingdom, and ${ }^{2}$ Department of Clinical Neurosciences and ${ }^{3}$ Behavioral and Clinical \\ Neuroscience Institute, University of Cambridge, Cambridge, CB2 3EB, United Kingdom
}

\begin{abstract}
Communication between the prefrontal cortex and subcortical nuclei underpins the control and inhibition of behavior. However, the interactions in such pathways remain controversial. Using a stop-signal response inhibition task and functional imaging with analysis of effective connectivity, we show that the lateral prefrontal cortex influences the strength of communication between regions in the frontostriatal motor system. We compared 20 generative models that represented alternative interactions between the inferior frontal gyrus, presupplementary motor area (preSMA), subthalamic nucleus (STN), and primary motor cortex during response inhibition. Bayesian model selection revealed that during successful response inhibition, the inferior frontal gyrus modulates an excitatory influence of the preSMA on the STN, thereby amplifying the downstream polysynaptic inhibition from the STN to the motor cortex. Critically, the strength of the interaction between preSMA and STN, and the degree of modulation by the inferior frontal gyrus, predicted individual differences in participants' stopping performance (stop-signal reaction time). We then used diffusion-weighted imaging with tractography to assess white matter structure in the pathways connecting these three regions. The mean diffusivity in tracts between preSMA and the STN, and between the inferior frontal gyrus and STN, also predicted individual differences in stopping efficiency. Finally, we found that white matter structure in the tract between preSMA and STN correlated with effective connectivity of the same pathway, providing important cross-modal validation of the effective connectivity measures. Together, the results demonstrate the network dynamics and modulatory role of the prefrontal cortex that underpin individual differences in inhibitory control.
\end{abstract}

Key words: diffusion MRI tractography; dynamic causal modelling; inferior frontal gyrus; presupplementary motor area; response inhibition; stop-signal task

\section{Introduction}

The ability to inhibit inappropriate or even dangerous actions is crucial for human behavior. Multistage neural pathways between the cortex, striatum, and the subthalamic nucleus (STN) have been proposed to support response inhibition through direct, indirect, and hyperdirect routes (Nambu et al., 2002; Redgrave et al., 2010; Wiecki and Frank, 2013). Two cortical regions are central to stopping motor actions: the presupplementary motor area (preSMA) and inferior frontal gyrus. For example, it is difficult to inhibit actions following lesions to the preSMA or

Received July 22, 2013; revised 0ct. 13, 2014; accepted 0ct. 16, 2014.

Author contributions: C.L.R. and J.B.R. designed research; C.L.R. and L.E.H. performed research; C.L.R., L.E.H., and J.B.R. analyzed data; C.L.R., M.C.A., and J.B.R. wrote the paper.

This work was supported by the Medical Research Council (MC-A060-5PQ30 and Doctoral Training Award to C.L.R.) and the Wellcome Trust (088324; J.B.R., L.E.H.). We thank M. Ewbank, R. Henson, and H. den Ouden for helpful discussion.

The authors declare no competing financial interests.

This article is freely available online through the J Neurosci Author Open Choice option.

Correspondence should be addressed to Dr Charlotte Rae, MRC Cognition and Brain Sciences Unit, 15 Chaucer Road, Cambridge, CB2 7EF, UK. E-mail: c.rae@bsms.ac.uk.

C. Rae's present address: Brighton and Sussex Medical School, Brighton, UK, and Sackler Centre for Consciousness Science, University of Sussex, Brighton, BN1 9RR, UK.

DOI:10.1523/JNEUROSCI.3093-13.2015

Copyright $\odot 2015$ Rae et al.

This is an Open Access article distributed under the terms of the Creative Commons Attribution License (http://creativecommons.org/licenses/by/3.0), which permits unrestricted use, distribution and reproduction in any medium provided that the original work is properly attributed. to the inferior frontal gyrus (Aron et al., 2003; Floden and Stuss, 2006; Nachev et al., 2007), and following interference via transcranial magnetic stimulation (TMS; Chambers et al., 2006; Cai et al., 2012). The STN is a key subcortical region for motor inhibition: lesions to the STN impair action stopping in rodents (Eagle et al., 2008), whereas stimulation of this region improves control of action in Parkinson's disease (Limousin and Martinez-Torres, 2008).

However, a clear model of how the preSMA and inferior frontal gyrus interact with the STN remains elusive (Aron et al., 2014). Neuroimaging suggests that, in response to stimuli indicating that a change in action is needed, the inferior frontal gyrus expedites a stopping process, implemented by pathways from the preSMA to subcortical regions such as the STN (Duann et al., 2009; Sharp et al., 2010; Zandbelt et al., 2013). However, electrophysiological evidence suggests that activity in the preSMA can precede activity in the inferior frontal gyrus during response inhibition. This implies a different architecture of network interactions, in which the inferior frontal gyrus executes the stop process (Neubert et al., 2010; Swann et al., 2012).

We have proposed that a fundamental property of the prefrontal cortex is that it can modulate connectivity between other brain regions, so as to reconfigure neural systems according to current task demands (Stephan et al., 2003; Rowe et al., 2007; Passingham et al., 2013). This raises the hypothesis that to successfully 

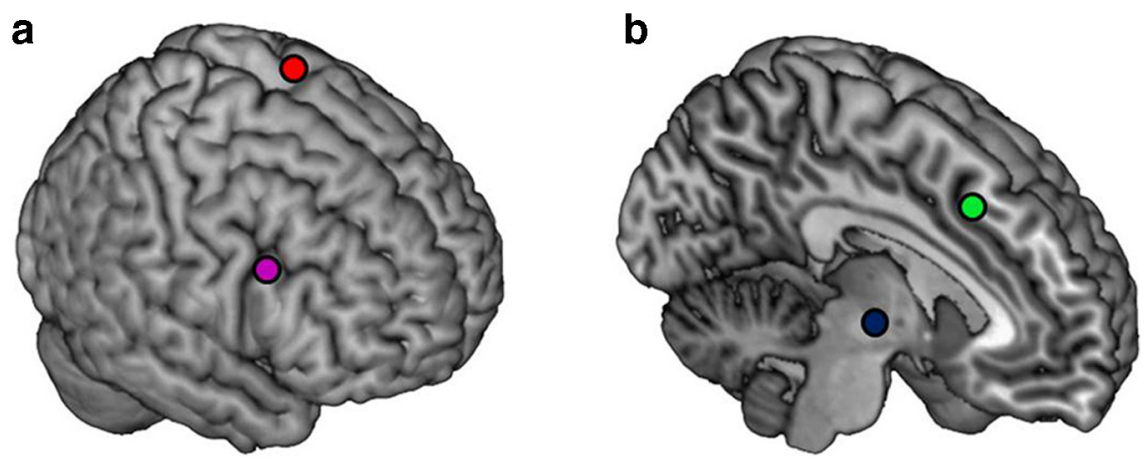

Figure 1. Lateral $(\boldsymbol{a})$ and medial $(\boldsymbol{b})$ views showing the regions included in the network models: right inferior frontal gyrus (pink), preSMA (green), STN (blue), left primary motor cortex (red).

inhibit a response, the inferior frontal gyrus or preSMA influence communication through subcortical pathways to the STN.

Therefore, we used functional neuroimaging to identify the interactions between critical cortical and subcortical areas during response inhibition. Healthy normal subjects underwent functional magnetic resonance imaging (fMRI) as they performed a manual stop-signal task (Logan et al., 1984). Using dynamic causal modeling (DCM; Friston et al., 2003) to estimate effective connectivity, we specified 20 generative models for the fMRI data that represented alternative hypotheses of the causal interactions between the inferior frontal gyrus, preSMA, and STN. By comparing the models' evidences, we identified the most likely model of prefrontal-subcortical interactions for response inhibition.

Next, we examined individual differences in behavior and brain structure (Cummins et al., 2012; Forstmann et al., 2012). An individual's ability to stop an action was predicted by structural connectivity between cortical and subcortical regions of the inhibitory control network, using diffusion-weighted imaging. This structural evidence provides powerful support for the significant correlations we also observed between the effective connectivity parameters and the efficiency of response inhibition.

\section{Materials and Methods}

Stop-signal task. The study was approved by the local research ethics committee and subjects gave informed written consent before participation. Sixteen healthy right-handed adults (age 20-38, mean 28; 12 males) performed a variant of the stop-signal task while being scanned with fMRI. In the stop-signal task (Logan et al., 1984), actions were either specified as single-finger presses of the right hand, or single-finger presses chosen by the subject (Rowe et al., 2010a; Rae et al., 2014). These two trial types were collapsed together for network analysis (see Dynamic causal modeling) given the lack of a significant difference in behavior or regional activations in this task (Rae et al., 2014). Subjects viewed a picture of a right hand, with a circle above each finger. Responses were cued by a color change of the circles to green. On $25 \%$ of trials, after a short variable delay, the green "go" cue changed to a red "stop" cue in conjunction with an auditory tone $(1000 \mathrm{~Hz}, 100 \mathrm{~ms})$, indicating that subjects should withhold their response. Staircase tracking algorithms modified the delay between go and stop cues in increments or decrements of $50 \mathrm{~ms}$, on a trial-by-trial basis, to maintain overall stopping accuracy close to $50 \%$ (including separate tracking algorithms for specified and chosen finger presses, but these did not significantly differ in the mean stop accuracy; Rae et al., 2014). Green "go" cues were presented for 1 s. An intertrial interval comprised continuous presentation of the hand picture, with no change in the gray background color of the circles. The stimulus onset asynchrony varied between 3 and $9 \mathrm{~s} \mathrm{(mean} 4.5 \mathrm{~s}$ ) to increase design efficiency. Subjects completed 432 go trials and 144 stop trials during continuous imaging, but divided into six blocks of equal length with a rest break of $20 \mathrm{~s}$ between blocks. Trial order was fully randomized. The stop-signal reaction time (SSRT) was used as the behavioral measure of stopping efficiency: a lower SSRT indicates less time is required to inhibit a response. SSRTs were calculated according to the integration method (Logan et al., 1984).

fMRI acquisition and preprocessing. 1450 BOLD-sensitive $\mathrm{T} 2{ }^{*}$-weighted echoplanar images were acquired on a 3T Siemens Trio, covering the whole brain (32 descending axial oblique slices $3 \mathrm{~mm}$ thick with $0.75 \mathrm{~mm}$ gap, in-plane resolution $3 \times 3 \mathrm{~mm}, \mathrm{TR}=2000 \mathrm{~ms}$, $\mathrm{TE}=30 \mathrm{~ms})$. The first five volumes were discarded to allow for steady-state magnetization. A high-resolution MPRAGE structural scan was acquired for registration and normalization $\left(1 \times 1 \times 1 \mathrm{~mm}^{3}\right.$ resolution, $\mathrm{TR}=2250$ $\mathrm{ms}, \mathrm{TE}=2.99 \mathrm{~ms})$. fMRI preprocessing and statistical modeling used SPM8, patch version r4667 (http://www.fil.ion.ucl.ac.uk/spm/) in MATLAB 7.8. Images were converted from dicom to nifti format, realigned to the mean image, and sinc interpolated in time to correct for slice timing differences during acquisition. The MPRAGE was coregistered to the mean echoplanar image using mutual information, and iteratively segmented and normalized to the SPM MNI152 template. The resulting normalization parameters were applied to the realigned and slice-time corrected echoplanar images, before smoothing with a Gaussian kernel of full-width halfmaximum $8 \mathrm{~mm}$.

A first level event-related analysis for each subject used a general linear model. Events were modeled with a duration of $1 \mathrm{~s}$ from trial onset, and convolved with the canonical hemodynamic response function. The design matrix was organized to separate driving inputs (e.g., for all trials) from the modulatory inputs (e.g., successful stopping) for DCM (Friston et al., 2003; Stephan et al., 2008), while accounting for all principal experimental variables across the six trial types (go-specified, go-select, stop-specified-correct, stop-specified-incorrect, stop-select-correct, and stop-select-incorrect) and nuisance terms (e.g., motion parameters). The first regressor represented the driving input of all trial types. The second, third, and fourth regressors were parametric modulators of trials. Parametric Modulator 1, "selection," comprised select $>$ specified trials (go-select, stop-select-correct, stop-select-incorrect $>$ go-specified, stop-specified-correct, stop-specified-incorrect). Parametric Modulator 2, "stopping," comprised correct stop $>$ go trials (stop-specified-correct, stop-select-correct $>$ go-specified, go-select). Parametric Modulator 3, "correct stopping $>$ incorrect stopping," comprised correct stop $>$ incorrect stop trials (stop-specified-correct, stop-select-correct $>$ stopspecified-incorrect, stop-select-incorrect). Parametric Modulators 1 and 3 were not used as driving or modulatory inputs for DCM, but were included in the design matrix to account for experimental variance. Go trials on which a subject made an error (for example, pressing with the wrong finger on a specified trial) were modeled in separate regressors, according to the error type (omission: RT > $1000 \mathrm{~ms}$, commission: RT < $100 \mathrm{~ms}$; error: wrong finger pressed). Finally, six nuisance regressors modeled subject movement as three translations and three rotations. Activations for correct response inhibition (stop-correct $>$ go) were examined using the stopping parametric modulator. A second-level SPM analysis used contrasts from the first level, with a one-sample $t$ test (see Fig. 2). Cluster-based FDR (FDRc) at $p<0.05$ was applied to correct for multiple comparisons (Chumbley and Friston, 2009). To extract regional time series for DCM, we specified an F test across the "all trials" regressor and three parametric modulators that were the first four columns of the design matrix ( $F$ contrast: $p<0.05$, uncorrected).

We extracted the first eigenvariate of the BOLD time series from four regions-of-interest (ROIs): left primary motor cortex (M1), STN, preSMA, and right inferior frontal gyrus (Fig. 1). For the M1, preSMA, and inferior frontal gyrus ROIs, we defined group peak coordinates from a second level group analysis, using the all trials column of the design matrix for M1 $(x=-34, y=-22, z=56)$, and the conjunction contrast of parametric modulators ( 1 , selection; 2 , stopping) for the preSMA and 
inferior frontal gyrus (preSMA: $x=-8, y=20, z=44$; inferior frontal gyrus: $x=48, y=14, z=28$ ). The inferior frontal gyrus peak corresponded to right superior pars opercularis (Aron and Poldrack, 2006; Hampshire et al., 2010; Verbruggen et al., 2010). For the M1, preSMA, and inferior frontal gyrus ROIs, we used each subject's $F$ test to identify local maxima closest to the group peak, conforming to appropriate anatomy (cf. Rowe et al., 2010b), and extracted the first eigenvariate from a 5 $\mathrm{mm}$ sphere at the subject-specific peak. Four subjects showed a relatively posterior activation at the border of SMA/preSMA ( $y=0$; Nachev et al., 2008).

For the STN ROI, we used a bilateral STN mask from the probabilistic maps described by Forstmann et al. (2012). Because the probabilistic maps do not correspond to masks of contiguous voxels, we smoothed the unthresholded left and right STN maps with a Gaussian kernel of $2 \mathrm{~mm}$ (original STN map voxel size: $0.5 \mathrm{~mm}$ ), using the function "fslmaths" in FSL (www.fmrib.ox.ac.uk/fsl). Then, using "fslmaths," the smoothed images were thresholded at 0.1 to produce left and right STN masks similar in spatial extent to the original STN maps, preserving the oblique $3 \mathrm{D}$ geometry of the nucleus, but composed of contiguous voxels. The left and right STN masks were combined. We applied this STN mask (total volume: 448 voxels at a resolution of $0.5 \mathrm{~mm}^{3}$ ) in each subject's $F$ test image to find the subject-specific peak within the STN region. We then extracted a $5 \mathrm{~mm}$ sphere eigenvariate at this location.

Dynamic causal modeling. We used DCM (Friston et al., 2003) to determine the most likely network with interactions between inferior frontal gyrus, preSMA, and the STN during successful response inhibition on the stop-signal task. DCM estimates the effective connectivity between brain regions according to (1) the average connections between the regions (DCM.A matrix), (2) modulatory influences on connections arising through experimental manipulations (namely successfully stopping an action; DCM.B matrix), and (3) condition-specific inputs that drive network activity (namely engaging in a response inhibition task; DCM.C matrix). If a model includes activity-dependent connections (that are modulated by regional activity, as opposed to condition-specific inputs), the model is equipped with a further set of $\mathrm{D}$ parameters, coupling regions (nodes) to connections. These models with a further set of parameters (the DCM.D matrix) are referred to as nonlinear DCMs, because the connectivity depends upon the square of activity.

In DCM, the neural dynamics of a model are mapped to the fMRI time series with a comprehensive forward model of the hemodynamic BOLD response. The DCM.A, DCM.B, and DCM.C (and DCM.D if specified) parameters of a model are estimated using Bayesian approximations to maximize the free-energy bound $(F)$ on the Bayesian model evidence. $F$ expresses the accuracy of a model given the data, adjusted for model complexity. $F$ is then compared across different models to determine the most likely generative model of the fMRI data.

We estimated 20 models that represented alternative hypotheses of interactions among four regions of interest: the inferior frontal gyrus, preSMA, STN, and primary motor cortex (Fig. 1). The choice of four regions was determined by the minimum set required to test our hypotheses of interactions between prefrontal cortex and the STN, extending previous neuroimaging and electrophysiological models (cf. Duann et al., 2009; Neubert et al., 2010; Sharp et al., 2010; Swann et al., 2012; Zandbelt et al., 2013). One could consider the inclusion of additional nodes, especially in the putamen, pallidum, and thalamus (cf. Frank, 2006; Redgrave et al., 2010; Wiecki and Frank, 2013). However, this is not necessary to determine the interactions between cortex and STN, because DCM paths implicitly represent both monosynaptic and polysynaptic connections between regions (Stephan et al., 2010).

We limited our models to the four regions necessary and sufficient to test our hypotheses regarding prefrontal-STN interactions and motor output. The structural architecture of these models was informed by primate anatomical connectivity. Specifically, tracer studies in macaques show that the preSMA connects to area 44, the STN, and M1, either directly or indirectly through the SMA (Picard and Strick, 1996; Inase et al., 1999; Nambu et al., 2002; Schmahmann and Pandya, 2006), whereas the STN connects indirectly to M1 via the globus pallidus and thalamus (Nambu et al., 2002). Note that DCM is agnostic as to whether connections between two specified regions are monosynaptic or polysynaptic.
We systematically varied the combinations of permitted (average) connections (DCM.A), modulatory effects of trial type (DCM.B), and driving inputs (DCM.C) across our set of 20 models to test alternative hypotheses of causal prefrontal-subcortical interactions during response inhibition (see Fig. 3). The set of 20 models were divided into "families" (Rowe et al., 2010b; Stephan et al., 2010) that shared average connectivity structure, namely whether connectivity between inferior frontal gyrus and preSMA was absent, unidirectional, or bidirectional (see Fig. 3; families A-D). In each model, average self-connections were applied to all four network nodes (cf. Rowe et al., 2010b). Across all 20 models, the inferior frontal gyrus and preSMA influenced the STN, either directly or indirectly, by modulation of cortical projections from each other. The STN did not feedback directly to the inferior frontal gyrus or preSMA, but fed-forward through the network via thalamus to motor cortex (Nambu et al., 2002).

The models within each family differed in site of modulatory input (see Fig. 3, dotted arrows). The modulatory input represented successful stopping (parametric modulator comprising correct stop $>$ go trials). We estimated 12 "linear" models (Friston et al., 2003), which varied in the prefrontal-STN connection site of modulatory input (DCM.B); and 8 "nonlinear" models (Stephan et al., 2008), in which the prefrontal region (inferior frontal gyrus or preSMA) serving as the site of driving inputs (in the DCM.C matrix) in turn gated the connection to the STN from the other prefrontal region (DCM.D matrix).

The driving input of a DCM, arising through condition-specific inputs, drives the network dynamics. Across all 20 models, driving inputs (DCM.C) represented engaging in the response inhibition task ("all trials" regressor of the design matrix including go, stop-correct, and stopincorrect trials). We applied driving inputs to both frontal regions (the inferior frontal gyrus and preSMA), in all 20 models. Driving inputs are represented by solid bold arrows in Figure 3 .

We used DCM10 in SPM8 (patch r4667) to estimate these 20 models for 16 subjects, estimating the free-energy bound on the model evidence (F). We compared the model evidences for the 20 models across the 16 subjects using Bayesian model selection (BMS; Stephan et al., 2009a) with fixed effects (see Fig. 4). This compares the group difference in $F$ for each model (which can also be expressed in terms of the group Bayes factor). BMS with fixed effects also provides the posterior model probability, which represents the probability that a given model generated the observed group data (range: $0-1$ ). We also performed BMS with a random-effects analysis, which accommodates differences in the generative models across subjects (Stephan et al., 2010). This provides the exceedance probability $(0-1)$, namely, the probability that a given model is more likely than any other model tested, in a group of subjects who may have generated data from different networks.

Using Bayesian parameter averaging (Stephan et al., 2009a), we estimated the connectivity values of the most likely model at the group level, across subjects (see Fig. 3). In each subject, we extracted the connectivity values of the most likely group model to test for correlations between individual differences in the strength of the prefrontal-STN interactions and SSRT, using Pearson correlations in SPSS 19.0 (IBM).

Diffusion-weighted MRI acquisition. Diffusion-weighted MRI data were acquired from the same subjects who participated in the fMRI stop-signal task, during the same scan session. Twice-refocused spinecho diffusion-weighted images were acquired with a voxel size of $1.8 \times$ $1.8 \times 2 \mathrm{~mm}$ (70 interleaved $2 \mathrm{~mm}$ axial slices; matrix size $106 \times 106$, field-of-view $192 \times 192, \mathrm{TR}=9300 \mathrm{~ms}, \mathrm{TE}=92 \mathrm{~ms}$ ). Diffusion weighting was applied along 64 gradient directions, at a $b$ value of $1000 \mathrm{~s} / \mathrm{mm}^{2}$. Two repetitions were acquired, with a volume with no diffusion weighting $\left(b=0 \mathrm{~s} / \mathrm{mm}^{2}\right)$ acquired at the beginning of each repetition.

Tractography between prefrontal cortex and STN. Preprocessing and probabilistic tractography analyses used FSL 4.1.8. Diffusion images were corrected for eddy currents and subject motion using "eddy_correct." Diffusion tensors were fitted using "dtifit." For tractography, "bedpostx" was used to model the diffusion distribution at each voxel, with two fibers modeled per voxel (default options; Behrens et al., 2007).

Masks of the right inferior frontal gyrus, right preSMA, and right STN were created in each subject's native T1 space. To aid in mask construc- 


\section{Stop-correct $>$ Go}
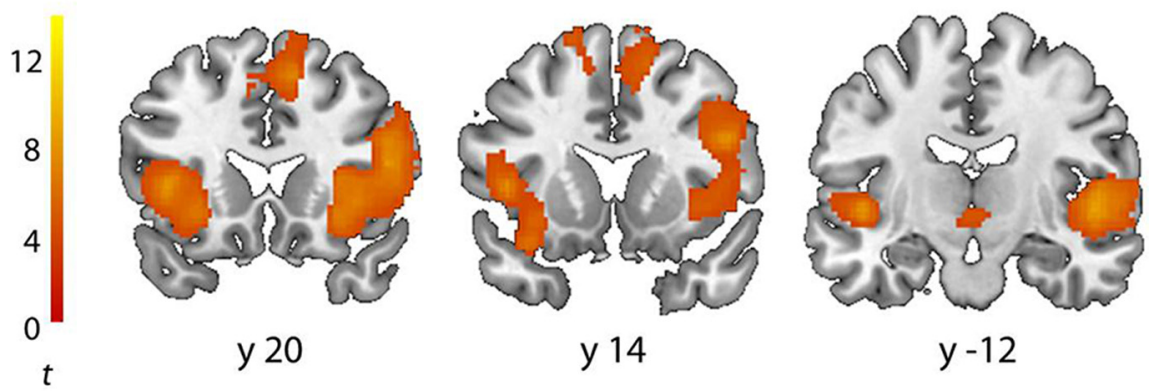

Figure 2. Group-level activations for correct-response inhibition (stop-correct $>$ go parametric modulator), thresholded at $p<0.05$ FDRc (Chumbley and Friston, 2009).

tion, each subject's structural MPRAGE image was segmented into gray matter, white matter, and CSF using FSL "fast," with default options.

For the inferior frontal gyrus masks, the BA44 and BA45 maps from the FSL Juelich histological atlas were transformed to subject structural space using "flirt." The masks were inspected in subject structural space and any voxels not meeting anatomical criteria for the inferior frontal gyrus were removed: this included voxels posterior to the precentral sulcus, superior to the fundus of the inferior frontal sulcus, inferior to the vertical ramus of the lateral fissure (at pars opercularis) or inferior to the horizontal ramus of the lateral fissue (at pars triangularis), and anterior to the point at which the horizontal ramus of the lateral fissure ended. Any voxels in gray matter or CSF were removed by subtracting the segmented gray matter and CSF voxels using "fslmaths," leaving only voxels corresponding to white matter underneath the inferior frontal gyrus.

For the preSMA masks, we used the combined preSMA+SMA map from the automatic anatomical labeling (Tzourio-Mazoyer et al., 2002) atlas. In the combined preSMA+SMA map, all voxels posterior to $y=0$ were removed, defined as the posterior border of the preSMA (Nachev et al., 2008). The preSMA map was transformed to subject native $\mathrm{T} 1$ space and any voxels in gray matter or CSF were removed by subtracting the segmented gray matter and CSF voxels using "fslmaths," leaving only voxels corresponding to white matter underneath the preSMA.

For the STN masks, we used the right STN mask created for STN fMRI time series extraction in the effective connectivity analysis, based on the STN maps described by Forstmann et al. (2012). The right STN mask was transformed to each subject's native T1 space using "flirt."

Using "probtrackx," we reconstructed tracts between (1) the right preSMA and right STN, and (2) the right inferior frontal gyrus and right STN (see Fig. 5). We chose to run tracts only in the right hemisphere due to the inclusion of the right inferior frontal gyrus region in the effective connectivity analysis (cf. Aron et al., 2003; Duann et al., 2009; Sharp et al., 2010). Diffusion MRI does not distinguish directionality of tracts, but results may differ slightly when different seeds are used. We therefore ran "probtrackx" twice: once with the cortical region applied as a seed and the STN as a target, and once with the STN applied as a seed and the cortical region as a target (default probtrackx options). An exclusion mask was applied at the midline, and the target mask was also set as a waypoint and as a termination mask.

The resulting output images for each tract ("fdt_paths") represent the most likely pathways that the sample streamlines take from the seed region. These were thresholded at $98 \%$ probability (i.e., only voxels showing at least 100/5000 streamlines were retained). The two separate probtrackx analyses for each tract were combined by adding the thresholded fdt_paths together with "fslmaths." Group-level tracts were created to remove spurious streamlines as follows: the thresholded tracts from each subject were binarized to remove the probabilistic connectivity values, and transformed to MNI space using FSL "applywarp." In MNI space, the tracts from each of the 16 subjects were summed and the group-level tracts thresholded such that only tract voxels present in at least $50 \%(8 / 16)$ subjects were retained. This procedure removes spurious streamlines (e.g., descending posteriorly from the STN to the cerebellum through the cerebellar peduncle; cf. King et al., 2012). Group-level tracts were binarized, and transformed to subject diffusion space using "flirt." In subject diffusion space, any tract voxels in gray matter were removed by thresholding the tracts at a fractional anisotropy threshold of $>0.2$.

Subjects' mean diffusivity images were then masked by the tracts and the average tract mean diffusivity extracted using "fslstats." We tested for correlations between SSRT and the mean diffusivity of (1) the right preSMA and right STN tract, and (2) the right inferior frontal gyrus and right STN tract, using Pearson correlations in SPSS 19.0 (IBM). Finally, we investigated the relationship between structural and effective connectivity directly. Specifically, we tested for Pearson correlations (SPSS) between (1) mean diffusivity in the tract between preSMA and STN, and the DCM.A parameter representing the average connectivity from the preSMA to the STN in the most likely model; and (2) mean diffusivity in the tract between inferior frontal gyrus and STN, and the DCM.D parameter representing nonlinear modulation in the most likely model (the aspect of the model most anatomically relevant for this tract).

\section{Results}

\section{Stop-signal task: behavior and activations}

The mean go reaction time was $627 \mathrm{~ms}$ (range: 528-723 ms, SD: $65 \mathrm{~ms}$ ). Mean stop accuracy (i.e., response correctly withheld) was 46\%. The mean SSRT was 296 ms (range: 227-355 ms, SD: $45 \mathrm{~ms}$ ).

Group-level activations for correct response inhibition (stopcorrect $>$ go parametric modulator) showed auditory and visual cortices, inferior frontal gyrus (pars opercularis and triangularis), insula, preSMA, premotor cortex, inferior parietal cortex, thalamus, and a region closely corresponding to the STN at $y=-12$ and $z=-4$ (thresholded at $p<0.05$ FDRc; Chumbley and Friston, 2009; Fig. 2; Table 1).

\section{Prefrontal-subcortical interactions during response inhibition}

We inverted 20 generative models representing alternative hypotheses of causal prefrontal-subcortical interactions during response inhibition (cf. Duann et al., 2009; Neubert et al., 2010; Sharp et al., 2010; Swann et al., 2012; Zandbelt et al., 2013). Using BMS (Stephan et al., 2009a), we compared the evidence for each model. The model comparison revealed striking results: there was very strong evidence in favor of one model above all others tested (see Fig. 4). In this nonlinear model, inhibiting a response was associated with an increase in connectivity from the preSMA to the STN. Crucially, however, the connectivity between the preSMA and STN was modulated by activity in the inferior frontal gyrus (Fig. 3b). There were also bidirectional corticocortical connections between the inferior frontal gyrus and preSMA (Fig. 3, family D).

Two model comparison measures demonstrated the superiority of this network architecture over the other models: the logmodel evidence (approximated by the free-energy of the model F; Fig. 4), and the posterior probability. The difference in log-model evidence between the most likely model and the nearest alternative was $>87$. To put this in context, a difference in log-model evidence of 5, equivalent to a Bayes factor of 150, is conventionally considered as very strong evidence for the more likely model 
Table 1. Local maxima of activations for correct-response inhibition (stopcorrect $>$ go parametric modulator), thresholded at $p<0.05$ (Chumbley and Friston, 2009), and localized according to the Anatomy toolbox in SPM8

\begin{tabular}{|c|c|c|c|c|c|}
\hline \multirow[b]{2}{*}{ Region } & \multirow[b]{2}{*}{ Hemisphere } & \multicolumn{3}{|c|}{ MNI coordinates } & \multirow[b]{2}{*}{$t$} \\
\hline & & $x$ & $y$ & $z$ & \\
\hline \multirow[t]{16}{*}{ Superior temporal gyrus } & $\mathrm{R}$ & 58 & -34 & 14 & 13.37 \\
\hline & $\mathrm{R}$ & 60 & -30 & 12 & 12.45 \\
\hline & $\mathrm{L}$ & -50 & -30 & 4 & 11.67 \\
\hline & $\mathrm{R}$ & 68 & -20 & 10 & 11.41 \\
\hline & $\mathrm{R}$ & 60 & -20 & 4 & 10.37 \\
\hline & $\mathrm{R}$ & 50 & -10 & 2 & 10.13 \\
\hline & $\mathrm{L}$ & -44 & -26 & 6 & 10.07 \\
\hline & $\mathrm{L}$ & -64 & -22 & 4 & 9.91 \\
\hline & $\mathrm{L}$ & -56 & -38 & 10 & 9.85 \\
\hline & $\mathrm{L}$ & -44 & -36 & 10 & 9.46 \\
\hline & $\mathrm{L}$ & -64 & -30 & 8 & 9.34 \\
\hline & $\mathrm{L}$ & -48 & -16 & 2 & 8.83 \\
\hline & $\mathrm{L}$ & -58 & -46 & 16 & 8.78 \\
\hline & $\mathrm{L}$ & -48 & -38 & 12 & 8.61 \\
\hline & $\mathrm{L}$ & -68 & -34 & 10 & 8.31 \\
\hline & $\mathrm{R}$ & 50 & -20 & -2 & 8.20 \\
\hline Supramarginal gyrus & $\mathrm{L}$ & -62 & -50 & 30 & 8.98 \\
\hline \multirow[t]{5}{*}{ Inferior frontal gyrus (pars triangularis) } & $\mathrm{R}$ & 52 & 22 & 14 & 8.32 \\
\hline & $\mathrm{L}$ & -32 & 30 & 2 & 8.24 \\
\hline & $\mathrm{L}$ & -42 & 18 & 6 & 8.23 \\
\hline & $\mathrm{R}$ & 46 & 16 & 24 & 7.75 \\
\hline & $\mathrm{L}$ & -40 & 26 & 0 & 7.13 \\
\hline \multirow[t]{7}{*}{ Fusiform gyrus } & $\mathrm{R}$ & 34 & -50 & -12 & 7.99 \\
\hline & $\mathrm{L}$ & -34 & -50 & -14 & 7.56 \\
\hline & $\mathrm{L}$ & -34 & -54 & -12 & 7.50 \\
\hline & $\mathrm{R}$ & 32 & -64 & -10 & 7.44 \\
\hline & $\mathrm{L}$ & -38 & -54 & -14 & 7.42 \\
\hline & $\mathrm{R}$ & 30 & -56 & -10 & 6.87 \\
\hline & $\mathrm{L}$ & -34 & -60 & -12 & 6.85 \\
\hline \multirow[t]{3}{*}{ Insula } & $\mathrm{R}$ & 30 & 28 & 4 & 7.97 \\
\hline & $\mathrm{R}$ & 38 & 26 & 2 & 7.95 \\
\hline & $\mathrm{L}$ & -30 & 18 & -8 & 6.50 \\
\hline Inferior frontal gyrus (pars opercularis) & $\mathrm{R}$ & 48 & 14 & 26 & 7.80 \\
\hline \multirow[t]{5}{*}{ SMA } & $\mathrm{R}$ & 10 & 20 & 50 & 7.14 \\
\hline & $\mathrm{R}$ & 16 & 12 & 60 & 5.10 \\
\hline & $\mathrm{L}$ & -8 & 18 & 54 & 4.26 \\
\hline & $\mathrm{L}$ & -6 & 22 & 50 & 4.07 \\
\hline & $\mathrm{R}$ & 10 & 14 & 70 & 3.99 \\
\hline \multirow[t]{3}{*}{ Inferior occipital gyrus } & $\mathrm{L}$ & -34 & -70 & -8 & 6.68 \\
\hline & $\mathrm{L}$ & -38 & -74 & -8 & 6.35 \\
\hline & $\mathrm{L}$ & -32 & -82 & -8 & 5.09 \\
\hline Calcarine sulcus (area 18) & $\mathrm{R}$ & 26 & -54 & 6 & 5.73 \\
\hline Cerebellum & $\mathrm{R}$ & 4 & -36 & -8 & 5.55 \\
\hline \multirow[t]{2}{*}{ Precentral gyrus } & $\mathrm{L}$ & -42 & 0 & 44 & 5.31 \\
\hline & $\mathrm{L}$ & -38 & -2 & 38 & 4.61 \\
\hline \multirow[t]{3}{*}{ Superior frontal gyrus } & $\mathrm{L}$ & -16 & 10 & 68 & 5.31 \\
\hline & L & -12 & 14 & 54 & 4.51 \\
\hline & $\mathrm{L}$ & -14 & 12 & 58 & 4.49 \\
\hline Parahippocampal gyrus & $\mathrm{R}$ & 18 & -42 & -8 & 5.27 \\
\hline \multirow[t]{3}{*}{ Middle occipital gyrus } & $\mathrm{R}$ & 36 & -88 & 6 & 4.95 \\
\hline & $\mathrm{R}$ & 36 & -84 & 14 & 4.34 \\
\hline & $\mathrm{R}$ & 30 & -90 & 5 & 4.23 \\
\hline \multirow[t]{2}{*}{ Calcarine gyrus } & $\mathrm{L}$ & -24 & -64 & 8 & 4.88 \\
\hline & $\mathrm{L}$ & -14 & -72 & 12 & 4.86 \\
\hline Precuenus & $\mathrm{R}$ & 16 & -72 & 42 & 4.83 \\
\hline Thalamus & $\mathrm{L}$ & -2 & -12 & -4 & 4.71 \\
\hline & $\mathrm{R}$ & 8 & -24 & -2 & 4.20 \\
\hline Cuneus & $\mathrm{L}$ & -14 & -74 & 36 & 4.55 \\
\hline & $\mathrm{R}$ & 20 & -62 & 38 & 4.07 \\
\hline Superior occipital gyrus & $\mathrm{L}$ & -18 & -78 & 26 & 4.55 \\
\hline & $\mathrm{L}$ & -16 & -80 & 30 & 3.97 \\
\hline Superior medial gyrus & L & -8 & 22 & 42 & 4.33 \\
\hline Inferior temporal gyrus & $\mathrm{R}$ & 44 & -60 & -10 & 4.17 \\
\hline
\end{tabular}

L, Left hemisphere; $\mathrm{R}$, right hemisphere; $x, y, z$ : coordinates of maximum activated voxel in standard MNI152 space; $t, t$ stat at this voxel.
(Raftery, 1995). The posterior probability for the most likely model was close to 1 (Fig. 4).

We also performed a complementary BMS using the randomeffects method, which accommodates differences in generative networks across subjects. The random-effects comparison also revealed very strong evidence in favor of the same response inhibition network, with an exceedance probability of 0.9 (maximum 1).

\section{Excitatory and inhibitory influences underlying response inhibition}

Having established the most likely prefrontal-STN network architecture underlying response inhibition, we then examined the interregional connections themselves, to clarify whether they were excitatory or inhibitory in nature (Fig. 3). The average connectivity parameter values (DCM.A matrix) represent how rapidly activity in one region influences activity in another region. A positive value indicates an increase in activity in the recipient region, namely, an excitatory influence from the source region, whereas a negative connectivity value indicates a decrease in activity, namely, an inhibitory influence from the source region. Likewise, the modulatory effects of a region in nonlinear models (reported in the DCM.D matrix) indicate an increase or reduction of the average connectivity between two regions. By examining whether these connectivity parameters were positive or negative, we can ascertain not just the existence of a causal relationship of one region on another, but establish the nature of that influence.

We used Bayesian parameter averaging to estimate the connectivity values of the most likely model at the group level, across subjects (Stephan et al., 2009a). The connection from the STN to M1 was inhibitory, in accordance with animal models in which the basal ganglia exert an inhibitory influence over thalamocortical structures (Redgrave et al., 2010). The preSMA to STN connection, and the modulatory influence of the inferior frontal gyrus, were excitatory. This indicates that when people successfully stop a motor response, the inferior frontal gyrus increases an excitatory influence of the preSMA on the STN, amplifying the downstream inhibition from the STN to the motor cortex (Fig. 3).

Thus, although the inferior frontal gyrus is central to implementing successful response inhibition (Aron et al., 2003; Chambers et al., 2006), these results suggest that the principal influence of this region on subcortical structures is positive. The inhibitory process that suppresses motor action likely happens at a subsequent stage in the pathway, and this inhibitory process is indirectly facilitated by the inferior frontal gyrus.

\section{Strength of network influences predicts response inhibition efficiency}

Examining the connectivity values of the model reveals how dynamics within the network architecture may give rise to the cancellation of a motor response. However, the strength of these network dynamics varies between people. We hypothesized that individual differences in the strength of prefrontal-STN interactions might relate to how well subjects are able to inhibit their responses on the stop-signal task. For example, people with greater increases in excitatory connectivity from the prefrontal regions to the STN on stop trials might be more efficient at cancelling their motor responses. The SSRT indicates how long subjects require to cancel their response after presentation of the stop cue. A lower SSRT indicates less time is required to cancel a motor response, and thereby more efficient response inhibition. 
a

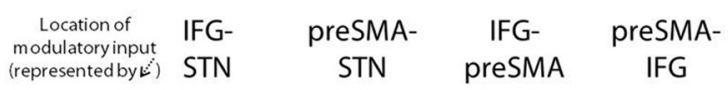

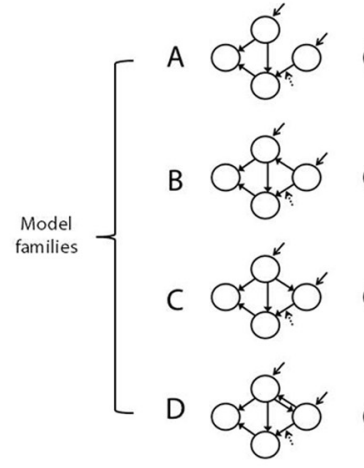

\section{LINEAR MODELS}
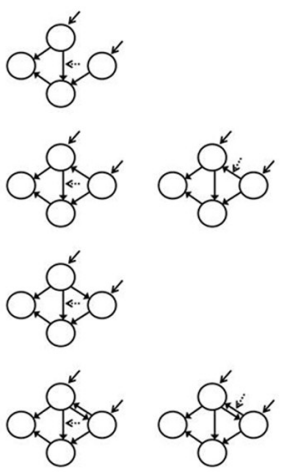

\section{NONLINEAR MODELS}

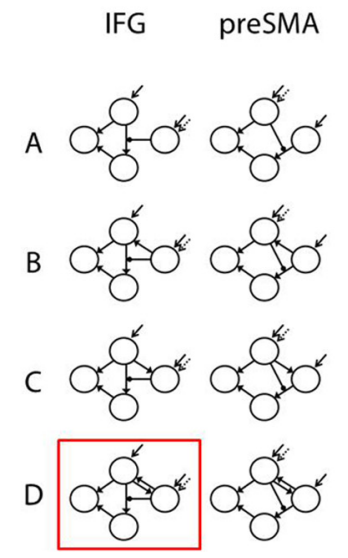

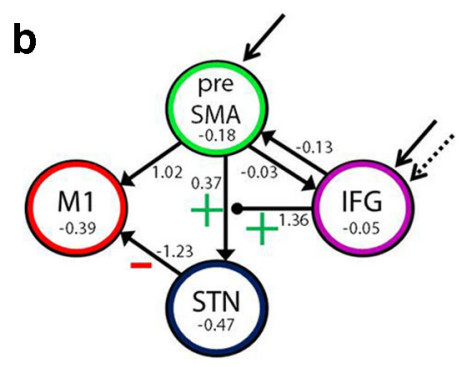

Figure 3. Structure of the 20 DCMs tested. $\boldsymbol{a}$, the four regions in the prefrontal-STN network are arranged schematically, in the same positions illustrated in $\boldsymbol{b}$. The 20 generative models represent alternative hypotheses of prefrontal-STN interactions during response inhibition. We compared 12 linear models and eight nonlinear models (see Materials and Methods). Dotted arrows indicate the modulatory effect of stopping (correct stop $>$ go). Solid bold arrows indicate driving inputs of task performance (all trials). Model families $(\boldsymbol{A}-\boldsymbol{D}$ ) share average connectivity structure between the inferior frontal gyrus and preSMA. The most likely model after BMS is highlighted, and shown in detail in $\boldsymbol{b}$ : response inhibition is associated with an increase in excitatory connectivity from the preSMA to the STN (DCM.A average connectivity). This connectivity is modulated by the inferior frontal gyrus (DCM.D nonlinear modulation), increasing the excitatory connectivity from the preSMA to the STN, and thereby amplifying the downstream inhibitory output from the STN to primary motor cortex. There are bidirectional connections between the inferior frontal gyrus and preSMA (Family D). Model connectivity values after Bayesian parameter averaging at the group level are shown inset (" + " increased excitatory connectivity, " - " increased inhibitory connectivity, at the group level across subjects).
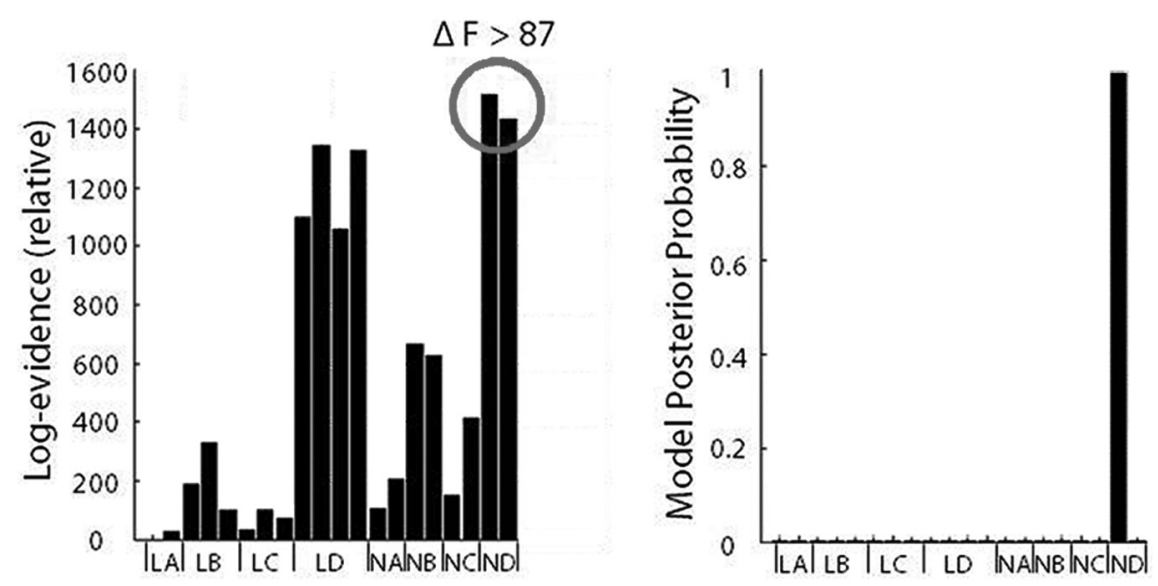

Figure 4. BMS. We compared models using the free-energy estimate of the log-model evidence $(F)$. There was very strong evidence in favor of one model (Fig. 3b). $\Delta F>5$ equates to a Bayes factor $>150$, and the most likely model has $\Delta F>87$, compared with the closest alternative, representing very strong evidence. The group posterior probability (close to 1 ) illustrates the superiority of the most likely model. "L" indicates a linear model, "N" a nonlinear model. Suffixes A-D indicate the model family. Within each model family, the models and their probability measures are arranged graphically in alphabetical order according to the location of the modulatory input.

To investigate the neural basis of individual differences in the ability to stop actions, we used Pearson correlations to compare the SSRT to the connectivity strength in the identified model. We examined the two critical pathways from the model: (1) the preSMA to STN connection (in DCM.A), and (2) the inferior frontal gyrus modulation of the effect of the preSMA (in DCM.D). Importantly, increases in both the excitatory preSMA to STN connectivity $(r=-0.524, p=0.019)$, and the excitatory inferior frontal gyrus modulation $(r=-0.482, p=0.029)$ predicted faster SSRTs, such that the greater the change in connectivity on stop trials (vs go trials), the more efficient a subject was at stopping actions. This indicates that the stronger the prefrontalSTN effective connectivity, the better subjects were at response inhibition. These correlations highlight that both preSMA-STN and inferior frontal gyrus-STN connectivity are significant influences on individual differences in people's capacity to stop actions.

\section{Structural prefrontal-subcortical connectivity predicts response inhibition efficiency}

Having established that differences in stopping ability relate to the strength of functional influences of the prefrontal regions on STN, we also sought converging evidence based on anatomical connectivity. The efficiency with which dynamic network interactions occur may be related to the structure of the white matter tracts carrying those interactions (Stephan et al., 2009b). Therefore, we determined whether the structure of prefrontal-STN white matter tracts was also a factor underlying individual differences in stopping efficiency, using diffusionweighted MRI.

We generated white matter tracts between (1) the preSMA and STN, and (2) the inferior frontal gyrus and STN, in the same subjects who performed the fMRI stopsignal task (Fig. 5). Because of the particular interest in righthemisphere lateralization of response inhibition (Aron et al., 2003; Duann et al., 2009; Sharp et al., 2010), and the inclusion of a right hemisphere inferior frontal gyrus region in our effective connectivity models, we focused on tracts in the right hemisphere. Mean diffusivity is a diffusion MRI-based marker of neural structure, and has been proposed to reflect tissue density, which in white matter may correspond to axonal count and myelination (Schmierer et al., 2007; Beaulieu, 2011). In white matter, lower mean diffusivity values reflect greater structural connectivity. We extracted the average mean diffusivity values from the two prefrontal-STN tracts in each subject. 


\section{a preSMA-STN}

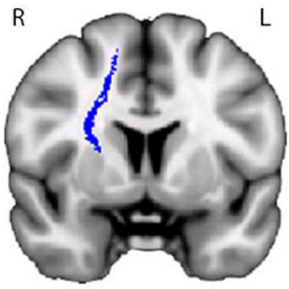

y 4

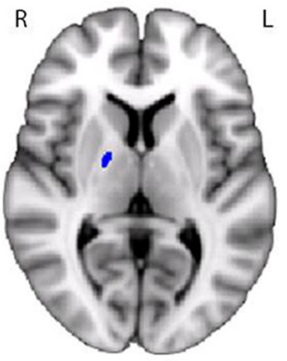

z 6

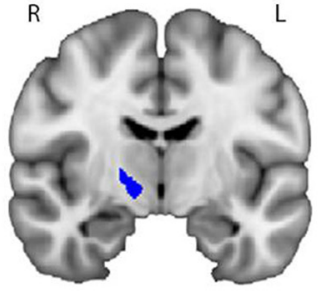

$y-10$

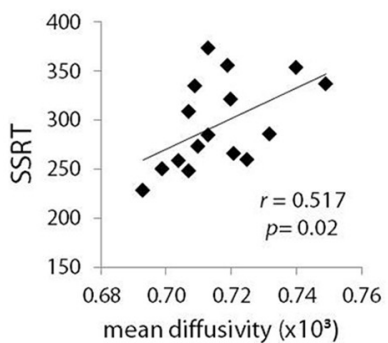

b IFG-STN
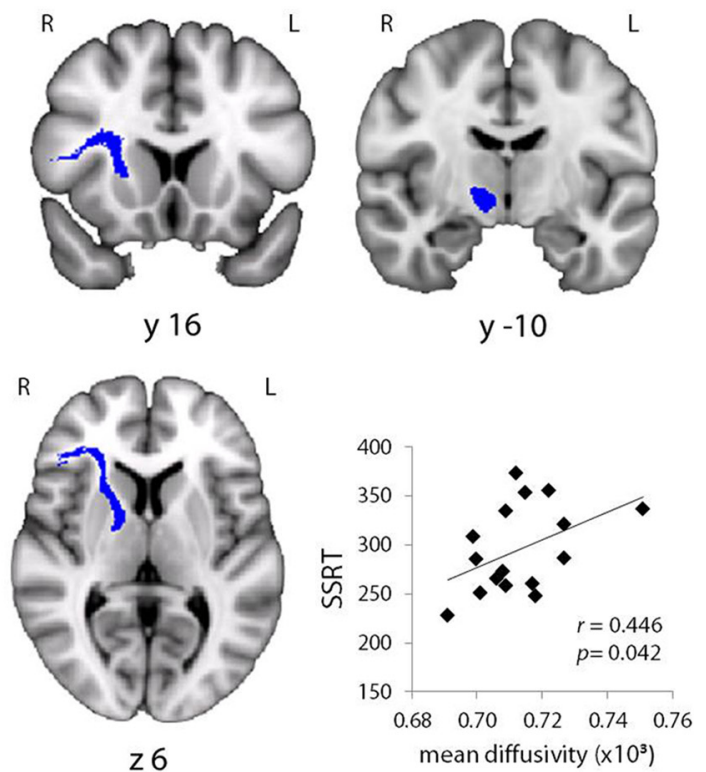

Figure 5. Prefrontal-STN white matter tracts. Using probabilistic tractography, white matter tracts were generated in the right hemisphere between ( $\boldsymbol{a}$ ) the preSMA and the STN, and ( $\boldsymbol{b}$ ) the inferior frontal gyrus and STN. Shown are the pathways present in at least $50 \%$ of subjects (blue) overlaid on the FSL MNI template. The average mean diffusivity (MD) was extracted from these tracts in each subject. There were significant positive correlations between SSRT and MD in ( $\boldsymbol{a}$ ) the preSMA and STN tract, and ( $\boldsymbol{b})$ the inferior frontal gyrus and STN tract, suggesting that the stronger the prefrontal-STN structural connectivity, the more efficient subjects were at inhibiting their responses on the stop-signal task (lower MD represents greater structural connectivity in white matter).

Critically, participants' SSRTs positively correlated with mean diffusivity in both (1) the preSMA and STN tract $(r=0.517, p=$ $0.020)$, and (2) the inferior frontal gyrus and STN tract $(r=$ $0.446, p=0.042$ ). Thus, the greater the prefrontal-STN white matter structural connectivity, the more efficient subjects were at inhibiting their responses, providing converging evidence with the effective connectivity analysis. Together, these results underscore the importance of prefrontal-STN connectivity in determining individual differences in the ability to stop actions.

\section{Structural connectivity predicts effective connectivity}

To investigate the relationship between structural and effective connectivity directly, we tested for correlations between mean diffusivity in the two prefrontal-STN tracts, and the most relevant DCM parameters of the most likely model. There was a significant negative correlation between mean diffusivity in the right preSMA and right STN tract, and the preSMA to STN connection (in DCM.A): this suggests that the lower the MD, the greater the effective connectivity $(r=-0.554, p=0.013)$. The correlation between mean diffusivity in the tract between right inferior frontal gyrus and STN, and the nonlinear modulation by inferior frontal gyrus of the effect of the preSMA on the STN (in DCM.D) was not significant $(r=-0.309, p=0.122)$.

\section{Discussion}

The prefrontal cortex was characterized by dynamic connectivity to other brain regions, and modulation of connectivity between other brain regions, according to task demands (Stephan et al., 2003; Rowe et al., 2007; Passingham et al., 2013). Specifically, we found that during response inhibition the inferior frontal gyrus modulated the excitatory influence of the preSMA on the STN, amplifying the downstream inhibition from the STN to the motor cortex. In healthy adults, individual differences in response inhibition were determined by variation in this neural network, in terms of both the dynamic changes in functional connectivity (from fMRI) and the network's anatomical connectivity (from diffusion-weighted imaging).

The inferior frontal gyrus, preSMA, and STN are key regions for motor inhibition (Aron et al., 2003; Floden and Stuss, 2006; Eagle et al., 2008), and are proposed to support the control of action in multistage direct, indirect, and hyperdirect pathways (Nambu et al., 2002; Wiecki and Frank, 2013). However, previous data on the interactions of the inferior frontal gyrus and preSMA in response inhibition were not able to resolve the pattern and nature of network interactions that exists between these regions. Lesions to either region can lead to deficits in stopping actions (Aron et al., 2003; Floden and Stuss, 2006), as can the application of TMS (Chambers et al., 2006; Cai et al., 2012). Paired-pulse TMS and electrocorticography studies suggested that activity in the preSMA may precede activity in the inferior frontal gyrus during response inhibition (Neubert et al., 2010; Swann et al., 2012). However, in contrast, whole-brain neuroimaging studies suggest that the inferior frontal gyrus may "lie upstream" from the preSMA: detecting stimuli that indicate a change in action is necessary, and then expediting a stop process from the preSMA to subcortical regions (Duann et al., 2009; Sharp et al., 2010; Zandbelt et al., 2013). Thus, whereas prior work clearly establishes the involvement of these brain structures in response inhibition, it could not establish the manner in which these regions work together to achieve this critical function of self-control.

The analysis of causal influences, using model evidences within DCM (Friston et al., 2003; Stephan et al., 2008), was ideally suited to resolve the alternative mechanisms of prefrontalSTN interactions for response inhibition. By comparing the generative causal models, each motivated by previous electrophysiological and neuroimaging data, we were able to determine which connections were significant contributors to the inhibition network, their direction, and their excitatory or inhibitory nature. The similarity of the results from fixed-and random-effects 
model selection analyses suggests that the identified model provides an excellent account of the organization and function of the inhibition network in healthy adults. In the most likely model, the inferior frontal gyrus increased the excitatory influence of the preSMA on the STN, amplifying the downstream inhibition from the STN to the motor cortex.

This model of prefrontal-STN interactions unifies the previous disparate results from neurophysiology and neuroimaging. It supports the neurophysiological evidence for the earliest activity in the preSMA during inhibition trials (Neubert et al., 2010; Swann et al., 2012) and the presence of corticocortical interactions between preSMA and inferior frontal gyrus (Zandbelt et al., 2013). It also demonstrates the functional importance of the inferior frontal gyrus (Aron et al., 2014) and its subcortical interaction with projections from the preSMA (Duann et al., 2009). Convergence of structural and functional connectivity correlations with behavior provides important validation of the conclusions of the fMRI analysis.

Diffusion-weighted MRI measures correlate with individual differences in the efficiency of response inhibition, including the white matter tracts between the inferior frontal gyrus, preSMA, and STN (Coxon et al., 2012; Forstmann et al., 2012). However, the histological features underlying these diffusion weighted imaging effects are not yet fully understood (Zatorre et al., 2012), and diffusion MRI cannot provide insights into the directionality of connections. In contrast, functional MRI data fitted to causal models, and compared in terms of model evidences, can enable reliable inferences about the significance and directionality of connections. Our results demonstrate the importance of both white matter tracts and functional interactions from the inferior frontal gyrus and preSMA to the STN for stopping ability.

Furthermore, we found evidence that the strength of dynamic functional network interactions was related to the structure of the white matter tracts carrying those interactions (cf. Stephan et al., 2009 b). There was a significant correlation between white matter structure in the preSMA and STN tract, and effective connectivity from the preSMA to the STN. The correlation between mean diffusivity in the right inferior frontal gyrus and right STN tract, and the nonlinear inferior frontal gyrus modulation, was not significant. However, the lack of a significant correlation needs to be interpreted with caution. Speculatively, it is possible that the modulatory influence of the inferior frontal gyrus is determined primarily by variance at synaptic terminals of the connections to the STN from the preSMA, rather than the integrity of connecting white matter tracts. However, the lack of a significant correlation may also be due to a contribution from other, indirect, white matter tracts. This is because DCM is agnostic to the anatomical route of connectivity, representing instead the sum of influences between regions. For this reason, we make no claim about the cortical regions (e.g., premotor cortex or SMA) or subcortical regions (striatum, globus pallidus pars interna and externa) which might contribute to the indirect influences of preSMA onto the STN. A third potential factor for the lack of a significant structure-function correlation is type II error, arising either from the presence of covariance of posterior model parameter estimates (Rowe et al., 2010b) or limited sample size.

In both structural and functional analyses, the connections converged on the STN, which has been proposed to act as a "brake," suppressing basal ganglia output until the appropriate course of action, such as inhibition of a response, is determined (Frank et al., 2007; Wiecki and Frank, 2013). In this study, our stop cue required subjects to stop all actions. In the context of such a braking role, the present results suggest that when excit- atory preSMA-to-STN connectivity and inferior frontal gyrus modulation is greater, STN activity increases more quickly, activating the brake function more rapidly. As a result, subjects with greater prefrontal-STN connectivity are more efficient at inhibiting their response and show a lower SSRT. A caveat is that DCM is agnostic to the monosynaptic or polysynaptic nature of the connections, and our model does not differentiate the direct, indirect and hyperdirect neuronal paths from cortex, via striatum and pallidum, to the STN (Redgrave et al., 2010; Stephan et al., 2010). Note also that inhibition of a response in response to prefrontal activation does not necessitate direct neurophysiological inhibition of motor neuronal ensembles (Aron et al., 2014).

In conclusion, we have shown that the ability of prefrontal cortex to react to behaviorally salient stimuli, and inhibit behaviors, arises from two interacting systems. First, the pathway from preSMA to STN, and second the facilitation of communication through this pathway by the inferior frontal gyrus. The function of these two interacting systems is in part genetically determined (Whelan et al., 2012) and can also be enhanced by pharmacological treatments (Ye et al., 2014a,b). We propose that the connectivity approach we present here provides a clear framework with which to determine the mechanistic origins of dysfunctional selfcontrol and impulsivity in clinical disorders.

\section{References}

Aron AR, Poldrack RA (2006) Cortical and subcortical contributions to stop signal response inhibition: role of the subthalamic nucleus. J Neurosci 26:2424-2433. CrossRef Medline

Aron AR, Fletcher PC, Bullmore ET, Sahakian BJ, Robbins TW (2003) Stopsignal inhibition disrupted by damage to right inferior frontal gyrus in humans. Nat Neurosci 6:115-116. CrossRef Medline

Aron AR, Robbins TW, Poldrack RA (2014) Inhibition and the right inferior frontal cortex: one decade on. Trends Cogn Sci 18:177-185. CrossRef Medline

Beaulieu C (2011) What makes diffusion anisotropic in the nervous system? In: Diffuson MRI: theory, methods and applications (Jones DK, ed). New York: Oxford UP.

Behrens TE, Berg HJ, Jbabdi S, Rushworth MF, Woolrich MW (2007) Probabilistic diffusion tractography with multiple fibre orientations: what can we gain? Neuroimage 34:144-155. CrossRef Medline

Cai W, George JS, Verbruggen F, Chambers CD, Aron AR (2012) The role of the right presupplementary motor area in stopping action: two studies with event-related transcranial magnetic stimulation. J Neurophysiol 108: 380-389. CrossRef Medline

Chambers CD, Bellgrove MA, Stokes MG, Henderson TR, Garavan H, Robertson IH, Morris AP, Mattingley JB (2006) Executive "brake failure" following deactivation of human frontal lobe. J Cogn Neurosci 18:444455. CrossRef Medline

Chumbley JR, Friston KJ (2009) False discovery rate revisited: FDR and topological inference using Gaussian random fields. Neuroimage 44: 62-70. CrossRef Medline

Coxon JP, Van Impe A, Wenderoth N, Swinnen SP (2012) Aging and inhibitory control of action: cortico-subthalamic connection strength predicts stopping performance. J Neurosci 32:8401-8412. CrossRef Medline

Cummins TD, Hawi Z, Hocking J, Strudwick M, Hester R, Garavan H, Wagner J, Chambers CD, Bellgrove MA (2012) Dopamine transporter genotype predicts behavioural and neural measures of response inhibition. Mol Psychiatry 17:1086-1092. CrossRef Medline

Duann JR, Ide JS, Luo X, Li CS (2009) Functional connectivity delineates distinct roles of the inferior frontal cortex and presupplementary motor area in stop signal inhibition. J Neurosci 29:10171-10179. CrossRef Medline

Eagle DM, Baunez C, Hutcheson DM, Lehmann O, Shah AP, Robbins TW (2008) Stop-signal reaction-time task performance: role of prefrontal cortex and subthalamic nucleus. Cereb Cortex 18:178-188. CrossRef Medline

Floden D, Stuss DT (2006) Inhibitory control is slowed in patients with right superior medial frontal damage. J Cogn Neurosci 18:1843-1849. CrossRef Medline 
Forstmann BU, Keuken MC, Jahfari S, Bazin PL, Neumann J, Schäfer A, Anwander A, Turner R (2012) Cortico-subthalamic white matter tract strength predicts interindividual efficacy in stopping a motor response. Neuroimage 60:370-375. CrossRef Medline

Frank MJ (2006) Hold your horses: a dynamic computational role for the subthalamic nucleus in decision making. Neural Netw 19:1120-1136. CrossRef Medline

Frank MJ, Samanta J, Moustafa AA, Sherman SJ (2007) Hold your horses: impulsivity, deep brain stimulation, and medication in parkinsonism. Science 318:1309-1312. CrossRef Medline

Friston KJ, Harrison L, Penny W (2003) Dynamic causal modelling. Neuroimage 19:1273-1302. CrossRef Medline

Hampshire A, Chamberlain SR, Monti MM, Duncan J, Owen AM (2010) The role of the right inferior frontal gyrus: inhibition and attentional control. Neuroimage 50:1313-1319. CrossRef Medline

Inase M, Tokuno H, Nambu A, Akazawa T, Takada M (1999) Corticostriatal and corticosubthalamic input zones from the presupplementary motor area in the macaque monkey: comparison with the input zones from the supplementary motor area. Brain Res 833:191-201. CrossRef Medline

King AV, Linke J, Gass A, Hennerici MG, Tost H, Poupon C, Wessa M (2012) Microstructure of a three-way anatomical network predicts individual differences in response inhibition: a tractography study. Neuroimage 59: 1949-1959. CrossRef Medline

Limousin P, Martinez-Torres I (2008) Deep brain stimulation for Parkinson's disease. Neurotherapeutics 5:309-319. CrossRef Medline

Logan GD, Cowan WB, Davis KA (1984) On the ability to inhibit simple and choice reaction time responses: a model and a method. J Exp Psychol Hum Percept Perform 10:276-291. CrossRef Medline

Nachev P, Wydell H, O'Neill K, Husain M, Kennard C (2007) The role of the pre-supplementary motor area in the control of action. Neuroimage 36 : T155-163. CrossRef Medline

Nachev P, Kennard C, Husain M (2008) Functional role of the supplementary and pre-supplementary motor areas. Nat Rev Neurosci 9:856-869. CrossRef Medline

Nambu A, Tokuno H, Takada M (2002) Functional significance of the cortico-subthalamo-pallidal "hyperdirect" pathway. Neurosci Res 43: 111-117. CrossRef Medline

Neubert FX, Mars RB, Buch ER, Olivier E, Rushworth MF (2010) Cortical and subcortical interactions during action reprogramming and their related white matter pathways. Proc Natl Acad Sci U S A 107:13240-13245. CrossRef Medline

Passingham RE, Rowe JB, Sakai K (2013) Has brain imaging discovered anything new about how the brain works? Neuroimage 66:142-150. CrossRef Medline

Picard N, Strick PL (1996) Motor areas of the medial wall: a review of their location and functional activation. Cereb Cortex 6:342-353. CrossRef Medline

Rae CL, Hughes LE, Weaver C, Anderson MC, Rowe JB (2014) Selection and stopping in voluntary action: a meta-analysis and combined fMRI study. Neuroimage 86:381-391. CrossRef Medline

Raftery A (1995) Bayesian model selection in social research. In: Sociological methodology (Marsden P, ed), pp 111-196. Cambridge, MA: Wiley-Blackwell.

Redgrave P, Rodriguez M, Smith Y, Rodriguez-Oroz MC, Lehericy S, Bergman H, Agid Y, DeLong MR, Obeso JA (2010) Goal-directed and habitual control in the basal ganglia: implications for Parkinson's disease. Nat Rev Neurosci 11:760-772. CrossRef Medline

Rowe JB, Sakai K, Lund TE, Ramsøy T, Christensen MS, Baare WF, Paulson OB, Passingham RE (2007) Is the prefrontal cortex necessary for establishing cognitive sets? J Neurosci 27:13303-13310. CrossRef Medline

Rowe JB, Hughes L, Nimmo-Smith I (2010a) Action selection: a race model for selected and non-selected actions distinguishes the contribution of premotor and prefrontal areas. Neuroimage 51:888-896. CrossRef Medline

Rowe JB, Hughes LE, Barker RA, Owen AM (2010b) Dynamic causal modelling of effective connectivity from fMRI: are results reproducible and sensitive to Parkinson's disease and its treatment? Neuroimage 52:10151026. CrossRef Medline

Schmahmann J, Pandya DN (2006) Fiber pathways of the brain. New York: Oxford UP.

Schmierer K, Wheeler-Kingshott CA, Boulby PA, Scaravilli F, Altmann DR, Barker GJ, Tofts PS, Miller DH (2007) Diffusion tensor imaging of post mortem multiple sclerosis brain. Neuroimage 35:467-477. CrossRef Medline

Sharp DJ, Bonnelle V, De Boissezon X, Beckmann CF, James SG, Patel MC, Mehta MA (2010) Distinct frontal systems for response inhibition, attentional capture, and error processing. Proc Natl Acad Sci U S A 107: 6106-6111. CrossRef Medline

Stephan KE, Marshall JC, Friston KJ, Rowe JB, Ritzl A, Zilles K, Fink GR (2003) Lateralized cognitive processes and lateralized task control in the human brain. Science 301:384-386. CrossRef Medline

Stephan KE, Kasper L, Harrison LM, Daunizeau J, den Ouden HE, Breakspear M, Friston KJ (2008) Nonlinear dynamic causal models for fMRI. Neuroimage 42:649-662. CrossRef Medline

Stephan KE, Penny WD, Daunizeau J, Moran RJ, Friston KJ (2009a) Bayesian model selection for group studies. Neuroimage 46:1004-1017. CrossRef Medline

Stephan KE, Tittgemeyer M, Knösche TR, Moran RJ, Friston KJ (2009b) Tractography-based priors for dynamic causal models. Neuroimage 47: 1628-1638. CrossRef Medline

Stephan KE, Penny WD, Moran RJ, den Ouden HE, Daunizeau J, Friston KJ (2010) Ten simple rules for dynamic causal modeling. Neuroimage 49: 3099-3109. CrossRef Medline

Swann NC, Cai W, Conner CR, Pieters TA, Claffey MP, George JS, Aron AR, Tandon N (2012) Roles for the pre-supplementary motor area and the right inferior frontal gyrus in stopping action: electrophysiological responses and functional and structural connectivity. Neuroimage 59: 2860-2870. CrossRef Medline

Tzourio-Mazoyer N, Landeau B, Papathanassiou D, Crivello F, Etard O, Delcroix N, Mazoyer B, Joliot M (2002) Automated anatomical labeling of activations in SPM using a macroscopic anatomical parcellation of the MNI MRI single-subject brain. Neuroimage 15:273-289. CrossRef Medline

Verbruggen F, Aron AR, Stevens MA, Chambers CD (2010) Theta burst stimulation dissociates attention and action updating in human inferior frontal cortex. Proc Natl Acad Sci U S A 107:13966-13971. CrossRef Medline

Whelan R, Conrod PJ, Poline JB, Lourdusamy A, Banaschewski T, Barker GJ, Bellgrove MA, Büchel C, Byrne M, Cummins TD, Fauth-Bühler M, Flor H, Gallinat J, Heinz A, Ittermann B, Mann K, Martinot JL, Lalor EC, Lathrop M, Loth E, et al. (2012) Adolescent impulsivity phenotypes characterized by distinct brain networks. Nat Neurosci 15:920-925. CrossRef Medline

Wiecki TV, Frank MJ (2013) A computational model of inhibitory control in frontal cortex and basal ganglia. Psychol Rev 120:329-355. CrossRef Medline

Ye Z, Altena E, Nombela C, Housden CR, Maxwell H, Rittman T, Huddleston C, Rae CL, Regenthal R, Sahakian BJ, Barker RA, Robbins TW, Rowe JB (2014a) Improving response inhibition in Parkinson's disease with Atomoxetine. Biol Psychiatry. Advance online publication. Retrieved October 12, 2014. CrossRef

Ye Z, Altena E, Nombela C, Housden CR, Maxwell H, Rittman T, Huddleston C, Rae CL, Regenthal R, Sahakian BJ, Barker RA, Robbins TW, Rowe JB (2014b) Selective serotonin reuptake inhibition modulates response inhibition in Parkinson's disease. Brain 137:1145-1155. CrossRef Medline

Zandbelt BB, Bloemendaal M, Hoogendam JM, Kahn RS, Vink M (2013) Transcranial magnetic stimulation and functional MRI reveal cortical and subcortical interactions during stop-signal response inhibition. J Cogn Neurosci 25:157-174. CrossRef Medline

Zatorre RJ, Fields RD, Johansen-Berg H (2012) Plasticity in gray and white: neuroimaging changes in brain structure during learning. Nat Neurosci 15:528-536. CrossRef Medline 